\title{
Estudo comparativo de interfaces tangíveis de programação voltadas a crianças da educação infantil
}

\author{
André Luís Alice Raabe ${ }^{1,2,3}$, Cesar Pereira Viana ${ }^{2}$, Julia Peron Metzger ${ }^{3}$ \\ ${ }^{1}$ Laboratório de Inovação Tecnológica na Educação \\ ${ }^{2}$ Mestrado em Computação Aplicada \\ ${ }^{3}$ Programa de Pós-Graduação em Educação \\ Universidade do Vale do Itajaí (UNIVALI) - Itajaí, SC - Brasil \\ raabe@univali.br, \{cesarviana, juliaperon\}@edu.univali.br
}

\begin{abstract}
Programmable toys are a way of bringing digital technologies into the classroom while avoiding the individual interaction characteristic of personal computers. However, there is no clarity as to how different interfaces of programmable toys influence children's learning in early childhood education. This work analyzed the interaction of children using two tangible programming interfaces for problem-solving. Most of the children did not reach the proposed goal, and all resolutions occurred through one of the interfaces. There are indications that the type of interface helps solve problems, but it is necessary to investigate if there is real impact on learning.
\end{abstract}

Resumo. Brinquedos programáveis são uma forma de trazer as tecnologias digitais para a sala de aula evitando a interação individual característica dos computadores pessoais. Entretanto, não há clareza a respeito de como diferentes interfaces dos brinquedos programáveis influenciam no aprendizado das crianças na educação infantil. Este trabalho analisou a interação de crianças com duas interfaces tangíveis de programação durante a resolução de problemas. A maior parte das crianças não alcançou o objetivo proposto, e todas as resoluções ocorreram por meio de uma das interfaces. Há indícios de que o tipo de interface auxilia na resolução de problemas, porém é necessário investigar se há impacto real no aprendizado.

\section{Introdução}

Os brinquedos de programar têm sido apresentados como uma alternativa para levar as tecnologias digitais até as salas de aula, eliminando a necessidade de deslocamento até um laboratório de informática [Raabe et al. 2015]. Além de proporcionar brincadeiras aliadas a estímulos cognitivos, esses brinquedos são programados de forma natural e colaborativa, evitando a individualidade característica da interação com computadores pessoais.

A interação com os brinquedos programáveis geralmente ocorre por meio de interfaces tangíveis ou TUIs - Tangible User Interfaces. Segundo Falcão e Gomes (2007), as interfaces tangíveis unem as vantagens da manipulação física a recursos computacionais e podem estimular sentidos e a inclusão de pessoas com deficiência. Estudos como de Horn et al. (2009), Sapounidis et al. (2015), Zuckerman e Gal-Oz (2013), Zhu et al. (2016), e Pugnali et al. (2017), apontam que as interfaces tangíveis 
VIII Congresso Brasileiro de Informática na Educação (CBIE 2019)

Anais dos Workshops do VIII Congresso Brasileiro de Informática na Educação (WCBIE 2019)

promovem maior colaboração e diversão do que as interfaces gráficas.

Apesar destas vantagens, não foi encontrado, nesses e em outros estudos, um foco nas características das interfaces tangíveis dos brinquedos programáveis. Por isso acredita-se que uma investigação sobre este tema pode representar uma contribuição para a área da Informática na Educação.

Este artigo apresenta um estudo exploratório inicial. É relatado o teste de duas interfaces tangíveis de um brinquedo programável. O teste foi realizado com crianças de 5 anos em um ambiente educacional infantil. Uma das interfaces são os botões do brinquedo e a outra são blocos de instruções. A hipótese é que as crianças têm diferentes desempenhos e reações durante a interação com diferentes tipos de interfaces tangíveis de programação.

O conceito de interfaces tangíveis é aprofundado na seção 2 . A seção 3 apresenta duas subseções com os dois tipos de interface utilizadas no teste. O método de aplicação é apresentado na seção 4. Por fim, são apresentados os resultados (seção 5) e as considerações finais e sugestões para trabalhos futuros (seção 6).

\section{Interfaces tangíveis}

Uma ideia inicial sobre as interfaces tangíveis é que são formas de interação que permitem o ato de manipular um objeto com as mãos. Essa forma de interação é mais intuitiva e adequada para criar experiências que encorajam autonomia e colaboração [Zuckerman e Gal-Oz 2013]. Além disso, a interação física e o realismo das interfaces tangíveis fazem com que sejam preferidas pelos usuários, causando maior estímulo e diversão [Zuckerman e Gal-Oz 2013].

Numa definição mais exata, porém, as fronteiras que definem o que são interfaces tangíveis podem ser bastante difusas [Falcão e Gomes 2007]. Teclado e mouse são TUIs? Diferentes classificações foram desenvolvidas com base nestes questionamentos.

Fishkin (2004) apresenta uma taxonomia que parte da seguinte observação: numa primeira etapa o usuário manipula a interface. Num segundo passo, ele observa o resultado. O quão distante o ponto de manipulação inicial está da expressão do resultado? Isso seria a "incorporação", a qual é dividida em quatro tipos:

- Completa: O ponto de manipulação é o mesmo que expressa o resultado. Um exemplo é o ábaco.

- Próxima: A expressão do resultado é próxima ao ponto de manipulação.

- Ambiental: A expressão de saída está "ao redor" do usuário. Um exemplo seria uma sala com projeção nas paredes.

- Distante: O ponto de manipulação interage com a expressão do resultado, que está em outro local. Um exemplo seria o controle remoto da televisão.

Horn e Bers (2019) focam sua classificação nas linguagens de programação tangíveis, separando-as em três categorias:

- Linguagens de Blocos Inteligentes: Seria o caso, por exemplo, de blocos físicos com componentes eletrônicos internos. Os blocos são capazes de armazenar as instruções e também executá-las. 
VIII Congresso Brasileiro de Informática na Educação (CBIE 2019)

Anais dos Workshops do VIII Congresso Brasileiro de Informática na Educação (WCBIE 2019)

- Linguagens de Demonstração Tangíveis: Neste caso o usuário demonstra ao dispositivo o que deve ser executado e ele então reproduz. A demonstração pode ocorrer por meio de sons, gestos ou entradas digitais. Alguns exemplos seriam bater palmas, empurrar o brinquedo, ou teclar em seus botões.

- Linguagens Externamente Compiladas: São representações simbólicas do programa a ser executado, porém cujos elementos não tem componentes eletrônicos embutidos. Blocos de madeira se encaixam nesta categoria. Há necessidade que um elemento externo capte e compile os símbolos.

As duas classificações apresentadas são úteis para definir as duas interfaces tangíveis usadas neste trabalho. Enquanto Fishkin (2004) foca na distância entre a entrada e a saída, Horn e Bers (2019) classificam a linguagem pela qual o usuário informa o programa ao dispositivo executor. Após a apresentação das interfaces (seções 3.1 e 3.2), elas são classificadas no Quadro 1.

\section{Interfaces tangíveis de um brinquedo programável}

Duas interfaces tangíveis foram testadas com uma turma de um Centro de Desenvolvimento Infantil (CDI). A primeira é o teclado do brinquedo RoPE - Robô Programável Educacional. Já a segunda é uma interface de programação em blocos que foi desenvolvida neste trabalho com base em outra interface existente.

As interfaces foram selecionadas por serem desenvolvidas no laboratório e devido ao baixo custo. As alternativas importadas - como Bee-Bot ${ }^{1}$, Mojobot $^{2}$, mTiny $^{3}$ - custariam, no mínimo, R\$ 800,00. As interfaces usadas são apresentadas com mais detalhes a seguir.

\subsection{O brinquedo de programar RoPE}

$\mathrm{O} \mathrm{RoPE}^{4}$ é um projeto que busca criar brinquedos de programar de baixo custo aderindo à realidade dos núcleos de educação infantil brasileiros [Raabe et al. 2015]. Atualmente já é um produto, disponível em Santa Catarina. O revestimento é de madeira cortada e gravada via cortadora a laser e seus botões são feitos via impressora 3D (figura 1). A principal funcionalidade do brinquedo é estimular o Pensamento Computacional das crianças, bem como a criatividade, o que é auxiliado pela possibilidade de personalizar a carcaça do brinquedo [Martins 2016].
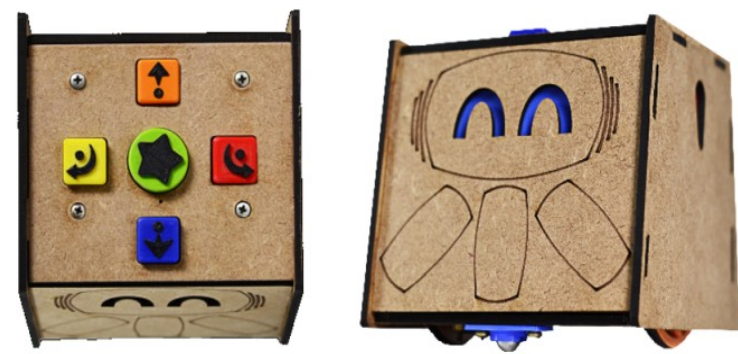

Figura 1: Brinquedo RoPE

1 https://www.terrapinlogo.com/beebot.html

$2 \mathrm{https}: / /$ www.mojobot.io

3 https://www.makeblock.com/mtiny

$4 \mathrm{https}: / / \mathrm{http}: / /$ constructoys.com/\#oque 
VIII Congresso Brasileiro de Informática na Educação (CBIE 2019)

Anais dos Workshops do VIII Congresso Brasileiro de Informática na Educação (WCBIE 2019)

A interação ocorre por meio de 5 botões (Figura 1), sendo eles: (1) girar para a esquerda (2) girar para a direita, (3) mover para frente, (4) mover para trás e (5) executar as instruções da memória ou limpar memória. Na execução de cada instrução é emitido um som característico, e também é emitida luz da mesma cor do botão gerador da instrução, de forma a haver uma tríade cor-movimento-som. Ao final da execução da sequência de comandos é tocado um som de finalização.

O brinquedo foi desenvolvido e evoluiu durante pesquisas realizadas com crianças. Pinheiro (2016) desenvolveu um sistema para criação de tapetes temáticos por sobre os quais o brinquedo se desloca. Martins (2016) explorou o uso do brinquedo para aprendizagem de habilidades de engenharia por crianças de três e quatro anos de idade. Esses trabalhos apontam que a atração das crianças pelo brinquedo já está confirmada. Já a interface de blocos, mostrada na próxima seção, é um protótipo em desenvolvimento e portanto com maior probabilidade de mau funcionamento ou inadequação à faixa etária que já teve contato com o brinquedo RoPE.

\subsection{Interface de programação em blocos}

A segunda interface usada neste trabalho é uma interface de programação em blocos. Denominada Blublocks ${ }^{5}$, é formada por um quadrado de madeira onde são encaixados blocos coloridos. Os blocos são os símbolos que representam os comandos a serem executadas por um brinquedo programável.

Neste caso, o brinquedo controlado pela Blublocks é o RoPE. Por isso há uma correspondência de cores e símbolos dos blocos com as cores e símbolos dos botões do brinquedo. A peça azul com seta para cima, por exemplo, significa mover para frente.

$\mathrm{Na}$ parte inferior de cada um dos quatro tipos de bloco (frente, trás, esquerda, direita) há uma marca fiducial, que é uma marca de fácil identificação por algoritmos de visão computacional. O celular, que fica embaixo da mesa transparente, capta as marcas através da câmera; identifica a sequência de peças; e então transmite a sequência ao brinquedo via Bluetooth.

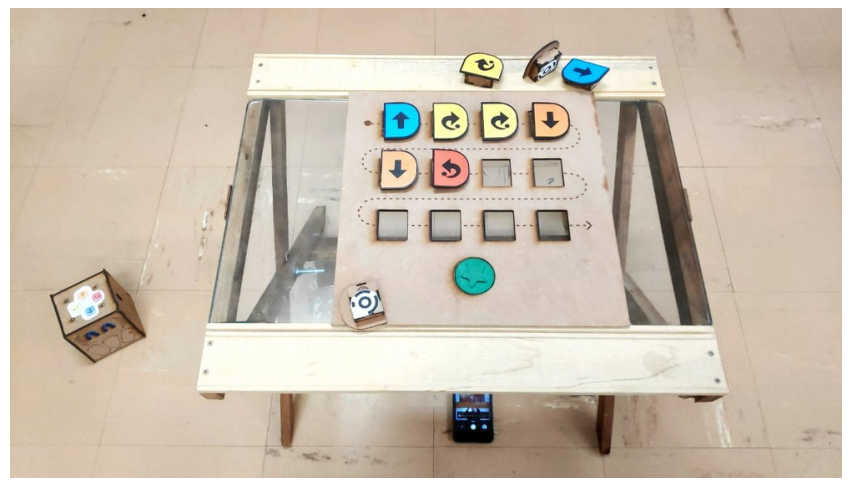

Figura 2: Interface de programação em blocos. Celular (embaixo da mesa) capta as marcas dos blocos (via câmera) e transmite ao RoPE (via Bluetooth).

Os botões da parte superior do RoPE destinado a ser controlado pela Blublocks foram removidos. A intenção é evitar tentativas de cliques nos botões do brinquedo durante a construção do programa com blocos. Para manter a referência de cores, no

5 O nome é inspirado no funcionamento com Bluetooth para a transmissão dos comandos. 
VIII Congresso Brasileiro de Informática na Educação (CBIE 2019)

Anais dos Workshops do VIII Congresso Brasileiro de Informática na Educação (WCBIE 2019)

lugar dos botões foi colado um papel com as mesmas cores e símbolos dos botões.

\subsection{Classificação das interfaces}

Após apresentadas as características das duas interfaces usadas neste trabalho, é possível relacioná-las com as duas classificações de TUIs citadas na seção 2. O Quadro 1 apresenta esse relacionamento:

Quadro 1: Classificação das interfaces utilizadas, segundo incorporação e tipo de linguagem

\begin{tabular}{|c|c|c|}
\hline & Teclado do brinquedo programável & $\begin{array}{c}\text { Interface de programação em } \\
\text { blocos }\end{array}$ \\
\hline $\begin{array}{c}\text { Horn e Bers } \\
\mathbf{( 2 0 1 9 )}\end{array}$ & $\begin{array}{c}\text { Linguagem de Demonstração } \\
\text { Tangível: usuário demonstra as ações } \\
\text { através de cliques }\end{array}$ & $\begin{array}{c}\text { Linguagem Externamente } \\
\text { Compilada: celular capta imagem } \\
\text { dos blocos e compila os comandos }\end{array}$ \\
\hline Fishkin (2004) & $\begin{array}{c}\text { Incorporação Completa: os botões } \\
\text { (entrada) estão unidos à saída (robô) }\end{array}$ & $\begin{array}{c}\text { Incorporação Distante: saída (robô) } \\
\text { está desacoplada da entrada } \\
\text { (blocos) }\end{array}$ \\
\hline
\end{tabular}

O teclado do brinquedo RoPE permite comunicar os comandos ao brinquedo através de uma linguagem de demonstração. A demonstração é feita através dos cliques nos botões do brinquedo. Na classificação de Fishkin (2004), o teclado é uma interface tangível de incorporação completa, pois está acoplado ao brinquedo programável.

Já a interface de blocos é uma linguagem externamente compilada, pois o celular capta a imagem das peças, gera a sequência de passos a serem executados e envia ao brinquedo. Essa interface é de incorporação distante, pois, enquanto o programa é criado sobre uma mesa, o brinquedo pode estar recebendo os comandos a metros de distância.

\section{Métodos}

Baseado em Sapounidis et al. (2019), o experimento foi planejado para ter duas fases. $\mathrm{Na}$ primeira fase, a pesquisadora tentou familiarizar as crianças com as duas interfaces. A segunda fase seria solicitar, às crianças, a resolução autônoma de desafios. Entretanto a familiarização exigiu um esforço cognitivo mais elevado do que o previsto e isso inviabilizou a interação autônoma.

As subseções seguintes caracterizam os participantes do experimento (seção 4.1), os materiais usados e o local do teste (seção 4.2) e a dinâmica de aplicação (seção 4.3).

\subsection{Participantes}

Em um Centro de Desenvolvimento Infantil da área urbana de Gaspar (Santa Catarina), 10 crianças participaram do estudo, sendo 5 do sexo feminino, 5 do sexo masculino. Todas as crianças tinham 5 anos de idade. As mesmas tiveram interação com o brinquedo de programar durante um experimento ocorrido um ano antes do teste. $\mathrm{O}$ número de participantes não é significativo estatisticamente, porém permite validar o funcionamento da interface de programação em blocos no ambiente real e investigar a hipótese levantada. 
VIII Congresso Brasileiro de Informática na Educação (CBIE 2019)

Anais dos Workshops do VIII Congresso Brasileiro de Informática na Educação (WCBIE 2019)

\subsection{Ambiente e materiais}

O experimento foi executado em uma sala reservada, característica de um ambiente infantil, com tapete emborrachado, uma mesa e bancos pequenos. As bolsas das crianças estavam penduradas na parede, demonstrando que aquele local lhes era familiar.

O ambiente, visível na figura 4, foi organizado de forma a conter as duas opções de interface. No tapete emborrachado foi posicionada uma cartolina verde quadriculada, com peças de encaixar no papel de obstáculos, formando o caminho Frente, Frente, Direita, Frente. O brinquedo RoPE foi colocado próximo à cartolina. Em frente à mesa foi posicionada a Blublocks experimental, e sobre a mesa uma cartolina azul quadriculada, também com obstáculos, formando o caminho Frente, Frente, Esquerda, Frente. Sobre a cartolina foi posicionado uma versão do RoPE com botões desenhados. Um notebook foi usado para filmar o experimento.

\subsection{Aplicação}

$\mathrm{O}$ experimento foi executado em conjunto à rotina comum de atividades do CDI. As 10 crianças estavam em uma sala adjacente e foram trazidas pela professora, a qual solicitou que sentassem no tapete. A pesquisadora saudou as crianças e estimulou a prestarem atenção em sua fala. As crianças negaram conhecer o brinquedo apesar de algumas já terem brincado em um experimento no ano anterior. A pesquisadora falou que o RoPE precisava encontrar uma tartaruga que estava perdida no "Labirinto da Floresta" (LF), e o "irmão do RoPE" precisava encontrar uma abelha que estava perdida no "Labirinto do Céu" (LC).

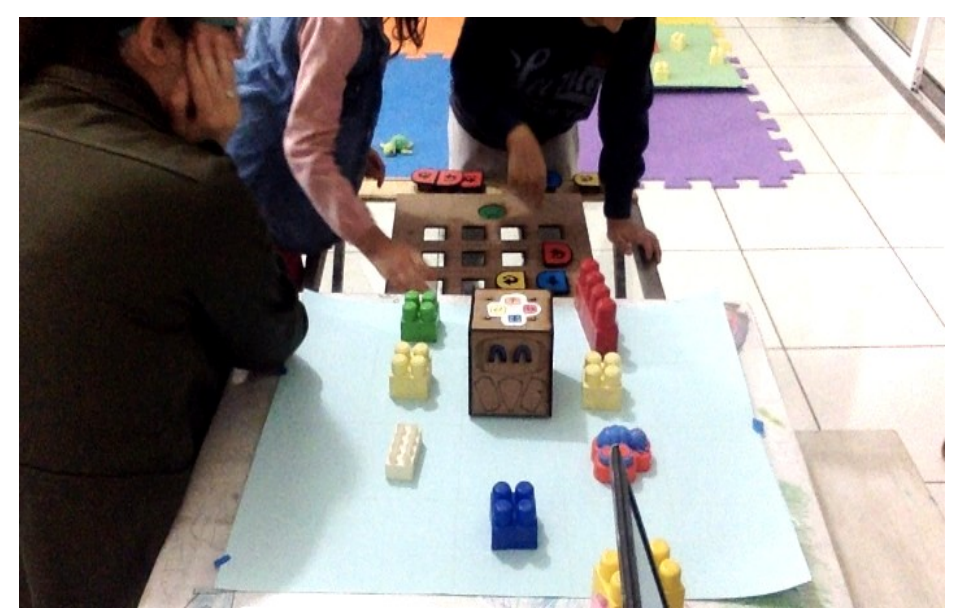

Figura 3: Labirinto do Céu (cartolina azul) e Labirinto da Floresta (cartolina verde, ao fundo).

Após a introdução, a turma retornou à sala adjacente para continuar suas atividades. A dinâmica ocorreu com a professora trazendo uma dupla de crianças por vez, formadas por uma criança de cada sexo. As três primeiras duplas fizeram a atividade na ordem LF-LC e as e com outras duas na ordem LC-LF. O tempo de interação foi de aproximadamente meia hora por dupla.

O processo ocorrido durante a interação com cada interface iniciou com a explicação do funcionamento pela pesquisadora. Colocando o brinquedo no chão, foram 
VIII Congresso Brasileiro de Informática na Educação (CBIE 2019)

Anais dos Workshops do VIII Congresso Brasileiro de Informática na Educação (WCBIE 2019)

programados alguns passos e feita a execução. Após isso o RoPE foi colocado no início de labirinto e foi solicitado às crianças que programassem o brinquedo para chegar até a tartaruga ou abelha. Na ocorrência de erros a pesquisadora reposicionava o brinquedo no início, porém sem programar nenhum passo para auxiliar a completar o desafio.

\section{Resultados}

O Quadro 2 demonstra, para cada uma das cinco duplas, se houve alcance do objetivo da tarefa proposta para cada um dos dois tipos de interface. Além disso a tabela mostra a ordem de aplicação das interfaces. É possível perceber que a maior parte não concluiu o objetivo, e aqueles que concluíram utilizaram a interface de programação em blocos. As duplas 1, 3 e 5 concluíram o objetivo usando a Blublocks e não concluíram usando o RoPE. As duplas 2 e 4 não tiveram sucesso com ambas as interfaces.

Quadro 2: Conclusões com sucesso por dupla e tipo de interface

\begin{tabular}{|l|l|l|l|}
\hline Dupla & Ordem de aplicação & Interface & Conseguiram executar a tarefa \\
\hline 1 & \multirow{2}{*}{ RoPE-Blublocks } & RoPE & Não \\
\cline { 3 - 4 } & & Blublocks & Sim \\
\hline 2 & \multirow{2}{*}{ RoPE-Blublocks } & RoPE & Não \\
\cline { 3 - 5 } & & Blublocks & Não \\
\hline 3 & \multirow{2}{*}{ RoPE-Blublocks } & RoPE & Não \\
\cline { 3 - 5 } & & Blublocks & Sim \\
\hline 4 & Blublocks-RoPE & Blublocks & Não \\
\cline { 3 - 5 } & & RoPE & Não \\
\hline 5 & Blublocks-RoPE & Blublocks & Sim \\
\cline { 3 - 5 } & & RoPE & Não \\
\hline
\end{tabular}

Uma possível explicação para o menor número de sucessos utilizando o RoPE, descrito no Quadro 2, é a necessidade de reprogramar os passos desde o início, dado que as instruções são eliminadas após a execução. Houve casos em que a dupla chegou próximo ao objetivo, porém errou completamente ao reprogramar. Utilizando a programação em blocos, foi possível simplesmente adicionar instruções faltantes ou alterar a sequência. A ordem de aplicação não parece influenciar o resultado, tendo em vista que houve sucessos e insucessos nas duas sequências possíveis.

A seguir são apresentadas verbalizações ocorridas durante as interações com as duas interfaces tangíveis. Bardin (1979) define análise de conteúdo como um conjunto de técnicas de análise das comunicações que permitem, por procedimentos sistemáticos, inferir conhecimentos relativos às condições de produção dessas mensagens. O Quadro 3 demonstra exemplos de habilidades exercitadas e as verbalizações ocorridas durante a interação com a mesa de programar e com o brinquedo. A comunicação entre crianças e a pesquisadora denota o exercício principalmente da colaboração e da depuração. As verbalizações foram possíveis devido à visualização do programa formado pelas peças. Isso vai ao encontro da ideia de Papert (1980, p. 21), que afirma a importância do "objeto-com-o-qual-pensar". 
VIII Congresso Brasileiro de Informática na Educação (CBIE 2019)

Anais dos Workshops do VIII Congresso Brasileiro de Informática na Educação (WCBIE 2019)

Quadro 3: Interação com a interface de programação em blocos

\begin{tabular}{|l|l|}
\hline Habilidade & Verbalização \\
\hline $\begin{array}{l}\text { Colaboração } \\
\text { (Blublocks) }\end{array}$ & Menina 1: [Fala ao menino] Vira pro outro lado, essa é pra frente! \\
\cline { 2 - 3 } & $\begin{array}{l}\text { Pesquisadora: Quer modificar o [programa] do [menino]? } \\
\text { Menina 2: Uhum! }\end{array}$ \\
\cline { 2 - 3 } & $\begin{array}{l}\text { Menino 2: Só uma [instrução]? } \\
\text { Menina 2: Não.. Tem mais! }\end{array}$ \\
\hline $\begin{array}{l}\text { Depuração } \\
\text { (Blublocks) }\end{array}$ & $\begin{array}{l}\text { Pesquisadora: Vocês conseguem ler o que vai acontecer? } \\
\text { Menino 1: Vai pra trás, pra trás, curva e pra frente. }\end{array}$ \\
\cline { 2 - 3 } & $\begin{array}{l}\text { Pesquisadora: Como vocês tinham me falado que tinha que fazer? } \\
\text { Menina 1: Duas pra frente, uma pro lado e uma pra frente! } \\
\text { Pesquisadora: E o que está aqui? } \\
\text { Menina 1: Duas pra frente, uma pro lado e uma pra frente! }\end{array}$ \\
\hline $\begin{array}{l}\text { Sequenciamento } \\
\text { (RoPE) }\end{array}$ & $\begin{array}{l}\text { Pesquisadora: O que tem que fazer primeiro pra ele chegar aqui? É o } \\
\text { que? } \\
\text { Menina 2: Tem que andar aqui, e andar aqui.. }\end{array}$ \\
\hline $\begin{array}{l}\text { Decomposição } \\
\text { (RoPE) }\end{array}$ & $\begin{array}{l}\text { Pesquisadora: Qual o passinho pra chegar até aqui? Um passinho de } \\
\text { cada vez } \\
\text { Menina 2: [aperta "frente" e "iniciar"] } \\
\text { Pesquisadora: E agora, pra ele girar pra cá? }\end{array}$ \\
\hline
\end{tabular}

A interação por meio dos botões do brinquedo ocorreu de forma individual, com cada criança criando um programa por vez. Já na programação em blocos alguns programas foram criados em conjunto, com uma criança iniciando e outra adicionando peças ou alterando as peças adicionadas.

As expressões faciais e gargalhadas observadas indicam que ambas as interfaces provocam engajamento. Nenhuma criança desistiu do experimento e finalizaram apenas com a indicação da pesquisadora. Perguntadas se queriam o retorno do RoPE ao CDI a resposta foi sim.

\section{Considerações finais e recomendações para trabalhos futuros}

Os dados coletados neste estudo inicial sugerem que, durante a interação com interfaces tangíveis, as características da interface podem influenciar na resolução de problemas. Nenhuma dupla de crianças teve sucesso usando a interface de botões, enquanto três duplas concluíram a atividade usando blocos de encaixar. Uma causa para isso pode ser a visualização das instruções nos blocos. A maior velocidade com que as instruções são inseridas via botões também pode reduzir o tempo de reflexão da criança a respeito do programa criado, diminuindo o número de sucessos.

Portanto, considera-se que há indícios favoráveis à hipótese de que as crianças têm desempenhos e reações diferentes durante a interação com diferentes interfaces tangíveis de um brinquedo programável. Quanto ao desempenho, todas as resoluções ocorreram por meio de uma das interfaces. Já as reações foram de engajamento e atração pelo brinquedo em ambas as interfaces. Na interface de botões, porém, algumas crianças apertavam apenas para ouvir o som produzido, sem refletir sobre o caminho programado. Enquanto isso, na interface de blocos, a orientação dos símbolos em 
relação ao ponto de vista causou estranhamento em algumas crianças. Uma criança observando a partir da esquerda considerou os símbolos de "esquerda" e "direita" como "trás" e "frente".

Algumas vulnerabilidades estão presentes neste estudo. Uma delas é o pequeno número de participantes e a homogeneidade dos mesmos. Uma pesquisa adicional deve trazer mais crianças e de idades variadas, como exemplo de Sapounidis et al. (2019), onde o intervalo foi de 6 a 12 anos. Além disso, o curto tempo de interação das crianças pode ter influenciado nos resultados. Uma pesquisa de médio ou longo prazo pode mensurar não apenas a influência das interfaces tangíveis no número de problemas resolvidos, mas também se houve real aprendizado dos conceitos de programação.

Com base nesta pesquisa, sugerimos que experimentos com interfaces tangíveis de brinquedos programáveis com crianças de 5 anos iniciem com o menor grau de dificuldade possível. Os resultados indicam que a resolução de problemas iniciando por um programa de quatro instruções envolvendo um giro apresentam um grau de complexidade inadequado para crianças de cinco anos.

\section{Referências}

Bardin, L. (1979). Análise de Conteúdo. São Paulo: Persona.

Falcão, T. P. e Gomes, A. S. (2007). Interfaces Tangíveis para a Educação. XVIII Simpósio Brasileiro de Informática na Educação, p. 579-589.

Fishkin, KennethP. (set 2004). A taxonomy for and analysis of tangible interfaces. Personal and Ubiquitous Computing, v. 8, n. 5.

Horn, M. e Bers, M. (2019). Tangible Computing. In: Fincher, S. A.; Robins, A. V. [Eds.]. . The Cambridge Handbook of Computing Education Research. 1. ed. Cambridge University Press. p. 663-678.

Horn, M. S., Solovey, E. T., Crouser, R. J. e Jacob, R. J. K. (2009). Comparing the use of tangible and graphical programming languages for informal science education. In Proceedings of the 27th international conference on Human factors in computing systems - CHI 09. . ACM Press. http://dl.acm.org/citation.cfm? doid=1518701.1518851, [acesso em Jun 1].

Martins, R. R. (2016). Desenvolvimento de uma metodologia para explorar habilidades de engenharia através do uso de brinquedos programáveis. Universidade do Vale do Itajaí.

Papert, S. (1980). Mindstorms: children, computers, and powerful ideas. New York: Basic Books.

Pinheiro, L. A. (2016). Análise da participação de crianças em atividades com brinquedos programáveis. Universidade do Vale do Itajaí.

Pugnali, A., Sullivan, A. e Umashi Bers, M. (2017). The Impact of User Interface on Young Children's Computational Thinking. Journal of Information Technology Education: Innovations in Practice, v. 16, p. 171-193.

Raabe, A., Rodrigues, A. J., Santana, A. M., et al. (26 out 2015). Brinquedos de Programar na Educação Infantil: Um estudo de Caso. . http://br-ie.org/pub/index.php/ wie/article/view/4985, [acesso em Jun 1]. 
VIII Congresso Brasileiro de Informática na Educação (CBIE 2019)

Anais dos Workshops do VIII Congresso Brasileiro de Informática na Educação (WCBIE 2019)

Sapounidis, T., Demetriadis, S., Papadopoulos, P. M. e Stamovlasis, D. (2019). Tangible and graphical programming with experienced children: A mixed methods analysis. International Journal of Child-Computer Interaction, v. 19, p. 67-78.

Sapounidis, T., Demetriadis, S. e Stamelos, I. (2015). Evaluating children performance with graphical and tangible robot programming tools. Personal and Ubiquitous Computing, v. 19, n. 1, p. 225-237.

Zhu, K., Ma, X., Wong, G. K. W. e Huen, J. M. H. (2016). How Different Input and Output Modalities Support Coding as a Problem-Solving Process for Children. In Proceedings of the The 15th International Conference on Interaction Design and Children - IDC '16. . ACM Press. http://dl.acm.org/citation.cfm? doid=2930674.2930697, [acesso em Abr 8].

Zuckerman, O. e Gal-Oz, A. (jul 2013). To TUI or not to TUI: Evaluating performance and preference in tangible vs. graphical user interfaces. International Journal of Human-Computer Studies, v. 71, n. 7-8, p. 803-820. 